\title{
Conversion of Unresectable to Resectable Liver Metastases from Colorectal Carcinoma Using Hepatic Arterial Chronomodulated Chemotherapy: A Case Report and Short Literature Review
}

\author{
Evgeny Y. Parnes', Maria S. Sayapina ${ }^{2 *}$, Alexey A. Tryakin'2, Mohamed Bouchahda ${ }^{3,4}$, \\ Pasquale F. Innominato ${ }^{3,4,5}$, Jean-Francois Morere ${ }^{3}$, Francis A. Levi3 ${ }^{3,4,5}$, Rene Adam ${ }^{4,6}$ \\ ${ }^{1}$ The Central Clinical Hospital of Civil Aviation, Moscow, Russia \\ ${ }^{2}$ N.N. Blokhin Russian Oncology Research Center, Moscow, Russia \\ ${ }^{3}$ Department of Medical Oncology, Assistance Publique Hôpitaux de Paris, Hôpital Paul Brousse, Villejuif, France \\ ${ }^{4}$ INSERM UMRS 776 "Biological Rhythms and Cancers”, Campus CNRS, Villejuif, France \\ ${ }^{5}$ Cancer Chronotherapy Team, Warwick Medical School, Coventry, United Kingdom \\ ${ }^{6}$ Hepato-Biliary Center, Assistance Publique Hôpitaux de Paris, Hôpital Paul Brousse, Villejuif, France \\ Email: *maria.sayapina@mail.ru
}

How to cite this paper: Parnes, E.Y., Sayapina, M.S., Tryakin, A.A., Bouchahda, M., Innominato, P.F., Morere, J.-F., Levi, F.A. and Adam, R. (2018) Conversion of Unresectable to Resectable Liver Metastases from Colorectal Carcinoma Using Hepatic Arterial Chronomodulated Chemotherapy: A Case Report and Short Literature Review. Surgical Science, 9, 358-366. https://doi.org/10.4236/ss.2018.910043

Received: August 29, 2018

Accepted: October 6, 2018

Published: October 9, 2018

Copyright $\odot 2018$ by authors and Scientific Research Publishing Inc. This work is licensed under the Creative Commons Attribution International License (CC BY 4.0).

http://creativecommons.org/licenses/by/4.0/

(c) (i) Open Access

\begin{abstract}
Background: The regional chronomodulated hepatic arterial infusion chemotherapy (HAIC) is an effective regimen for the treatment of patients with unresectable liver metastases from colorectal cancer, especially for the conversion into resectability. Aim: To demonstrate that chronomodulated HAI triplet chemotherapy according to OPTILIV protocol is well tolerated and displayed high antitumor activity in this heavily-pretreated patient. Case Presentation: A 54 years old patient from Russia was treated for a tumor in the ascending colon presented with 13 hepatic metastases ranging from 0.3 to $2.7 \mathrm{~cm}$ in diameter. He underwent a laparoscopic right hemicolectomy, 12 cycles of FOLFIRINOX combined to bevacizumab for the last 5 cycles, resulting in a partial response according to CT scan. It was decided to perform a two-stage hepatectomy at Paul Brousse hospital: left partial hepatectomy allowed the excision of 9 lesions. Radio frequency ablation was performed in 2 nodular lesions. Afterwards, the patient received 5 cycles of chronomodulated triplet chemotherapy into the hepatic artery, according to the OPTILIV protocol design, yet without cetuximab, because of the KRAS mutation in the liver metastases, with a partial response. The patient could then undergo the second stage of the planned right hepatectomy, which turned out to be an R0
\end{abstract}


resection followed by receiving three courses of chronomodulated HAIC. Disease progression was documented after 3 months. Chronomodulated FOLFIRI chemotherapy was re-started intravenously, in combination with Aflibercept and it was associated with further disease progression. The genetic analysis of our patient's cancer revealed a high level of MSI. The patient was included in the Phase 2 CheckMate- 142 trial and received nivolumab 3 $\mathrm{mg} / \mathrm{kg}$ every 2 weeks within 3 months. Treatment was discontinued due to ineffectiveness. Then the patient underwent radiotherapy geared towards reduction of pain. Afterwards, the patient died from the disease progression 2 years after the beginning of treatment. Conclusion: In this article, the authors report a clinical case with chronomodulated HAIC as rescue therapy in a heavily pretreated patient with metastatic colorectal cancer, allowing to achieve an objective response despite prior progression on FOLFIRINOX (the same triplet chemo by IV route). This strategy permitted to overcome drug resistance and to perform further complete resection of the liver metastases with prolonged patient survival. Thus, chronomodulated HAI is useful in patients with liver metastases from colorectal cancer and deserves to be further assessed prospectively in clinical trials chemotherapy.

\section{Keywords}

The Regional Chronomodulated Hepatic Arterial Infusion Chemotherapy, Metastatic Colorectal Cancer, Conversion of Unresectable to Resectable Liver Metastases

\section{Introduction}

Worldwide, liver metastases develop in nearly $60 \%$ of the patients with colorectal carcinoma [1]. The vast majority $(80 \%-90 \%)$ of patients present with unresectable liver metastases, and 5-year survival rate in these patients can range from $25 \%-35 \%$ [2] [3]. The potential value of hepatectomies for achieving long-term survival in patients with initially non-resectable colorectal liver metastases (CLM) has resulted in the development of liver-targeted oncological strategies [4]. Hepatic-arterial infusion chemotherapy (HAIC) has been developed to ensure a greater locoregional exposure to cytotoxic agents. HAIC induces significantly higher response rates (RR) than systemic chemotherapy [5] [6] [7] [8] [9]. A 5-year survival rate of $33 \%$ has been achieved after conversion hepatectomy and cure could be achieved in $16 \%$ of patients with initially unresectable CLM after effective downsizing chemotherapy [10]. Such liver-directed chemotherapy could benefit further from adjustment of drug-delivery pattern to the circadian clock in healthy hepatocytes [11]. Nevertheless, a large, randomized trial as well as a meta-analysis of three randomized trial comparing chronotherapy vs. conventional delivery of oxaliplatin-5-fluorouracil-leucovorin using individual dose escalation revealed a survival advantage in men but not in women. These results suggested the deleterious effect of dose intensification on 
the circadian rhythms of women, for whom chronotherapy needed to be personalized [12] [13]. The OPTILIV (Optimal Control of Liver Metastases from Colorectal Cancer with Cetuximab and Hepatic Artery Infusion of Chemotherapy) trial was the first prospective international trial, whose primary end point was the conversion of initially uncontrolled and/or unresectable CLM from colorectal cancer to resection, employing an aggressive liver-specific medico-surgical strategy [14] [15]. We reasoned that the triplet HAI could represent the most effective regimen for CLM downsizing, and enable adequate systemic treatment for preventing extrahepatic dissemination in previously heavily-pretreated patient, yet without cetuximab, because of the KRAS mutation in the liver metastases. In the present case, we determined early response according to RECIST criteria, qualifying early response as an objective response after 5 treatment courses, resulting in R0 resection and a better overall survival. The aim of this case report is to summarize the available evidence regarding optimal therapeutic strategies for converting initially unresectable metastases for potentially curative resection.

\section{Clinical Case}

A 54 years old patient from Russia was treated for a tumor in the ascending colon presented with 13 hepatic metastases ranging from 0.3 to $2.7 \mathrm{~cm}$ in diameter, initially in Moscow. He underwent a laparoscopic right hemicolectomy at N.N. Blokhin Russian Oncology Research Center on November 26, 2013. The tumor was histopathologically diagnosed as poorly differentiated adenocarcinoma which invades through the muscularis propria into the pericolorectal tissues. Metastatic lymphadenopathy was also noted in 1 regional lymph node. No cancer cells were found at the outer edge. Staging therefore classes the adenocarcinoma as being stage IV (T3N1M1a). Conclusion of genetic analysis: 1 . In the sample of the primary tumor, but not in a metastatic lymph node, a high level of microsatellite instability was found. In the exon 4 of the KRAS gene, a mutation p.A $146 \mathrm{~T}$ was found. Preoperative levels were normal for carcinoembryonic antigen (CEA), but not for carbohydrate antigen 19-9 (CA19-9, $8500 \mathrm{U} / \mathrm{mL}$; upper limit of normal of $38 \mathrm{U} / \mathrm{mL}$ ).

Afterwards, the patient received 12 cycles of FOLFIRINOX (IV Irinotecan 180 $\mathrm{mg} / \mathrm{m}^{2}$, Oxaliplatin $85 \mathrm{mg} / \mathrm{m}^{2}$, Fluorouracil bolus $400 \mathrm{mg} / \mathrm{m}^{2}$, Fluorouracil infusion $2400 \mathrm{mg} / \mathrm{m}^{2}$ ) combined to bevacizumab for the last 5 cycles, resulting in a partial response according to CT scan (Figure 1). Furthermore, CA19-9 decreased from $3500 \mathrm{U} / \mathrm{mL}$ postoperatively to $171 \mathrm{U} / \mathrm{mL}$ on FOLFIRINOX. This treatment however induced grade 3 diarrhea, grade 4 neutropenia and grade 1 neurotoxicity after the $7^{\text {th }}$ course.

In this context, it was decided to perform a two-stage hepatectomy at Paul Brousse hospital: the first one, left partial hepatectomy allowed the excision of 9 lesions on July 15, 2014. Radio frequency ablation was performed in 2 nodular lesions in segments IV and VIII, and in one lesion in segment II. Histopatholog- 
ic assessment revealed 50\% tumor, $20 \%$ necrosis, $10 \%$ fibrosis and $20 \%$ non tumorous in the liver metastases. Molecular analysis showed the presence of mutation A146T p. Ala 146Thr (c.436G > A) in the exon 4 and L19FpLeu19Phe $(\mathrm{C} 57 \mathrm{G}>\mathrm{T})$ in the exon 2 of the KRAS gene.

On July 29 CA19.9 level increased from 171 to $2000 \mathrm{U} / \mathrm{mL}$, indicating rapid tumour progression (at the same time with the growth of liver lobe metastases confirmed by MRI). It was then decided to administer chronomodulated triplet chemotherapy into the hepatic artery, according to the OPTILIV protocol design, yet without cetuximab, because of the KRAS mutation in the liver metastases. An indwelling hepatic arterial catheter (HAC) with an access port was inserted. 5 -FU $\left(2800 \mathrm{mg} / \mathrm{m}\left({ }^{2}\right)\right)$ was delivered in combination with irinotecan $(180$ $\left.\mathrm{mg} / \mathrm{m}\left({ }^{2}\right)\right)$ and oxaliplatin $\left(85 \mathrm{mg} / \mathrm{m}\left({ }^{2}\right)\right)$ through a HAC connected to a multichannel, programmable pump every 14 days (Melodie, Aguettant, France) [14] [15]. The patient underwent 5 cycles. This combination permitted the normalization of the CA19.9 levels, and achieved a partial response in second line, with the number of the right liver lobe metastases decreasing from 11 to 4, while allowing for a considerable liver regeneration, as evaluated with MRI. The patient could then undergo the second stage of the planned right hepatectomy on October 30, 2014, which turned out to be an R0 resection. The histological results showed evidence of metastatic hepatic colloidal mucus adenocarcinoma $(<5 \%)$ with safe margins. The patient then continued receiving three courses of chronomodulated triplet chemotherapy into the hepatic artery after hepatectomy (November 27, December 11, and December 22, 2014), with medium tolerance, including thrombocytopenia and elevated gamma-glutamyl transferase levels. Local thrombolytic therapy for hepatic artery thrombosis following chemotherapy infusion catheter placement was also needed.

Disease progression was documented on January 12,2015 , as shown with both an increase of CA 19-9 from 28 to $294 \mathrm{U} / \mathrm{mL}$, and the appearance of two bone metastases on the scanografictest, one on the level of 3 right ribs and the other one on the left ischial pubic bone. PET FDG (positron emission tomography) showed hypermetabolic heterogeneous lesions in a residual segment from the right hepatectomy, three months after surgery, i.e. on January 20, 2015. PET FDG also revealed a hypermetabolic diaphragmatic lesion near the site of the right hepatectomy, as well as hypermetabolic lesions of peritoneal carcinomatosis, lymphatic, left pulmonary, pleural, bone and muscular areas. The patient however only complained of pelvic and rib bone pains and some joint pain. Chronomodulated FOLFIRI chemotherapy was re-started intravenously, in combination with Aflibercept (antiangiogenic targeted therapy), with courses to be spaced by 21 days. Yet this third line treatment in this patient with very advanced disease was complicated by diarrhea, low blood counts, mouths sores and nasal bleeding and it was associated with further disease progression.

The genetic analysis of our patient's cancer revealed a high level of MSI and tumor heterogeneity (Table 1). The patient was included in the Phase 2 Check- 
Mate-142 trial evaluating nivolumab alone or in combination with ipilimumab in patients with previously treated metastatic colorectal cancer, including those with MSI-H. The patient received nivolumab $3 \mathrm{mg} / \mathrm{kg}$ every 2 weeks from March until May 2015. Treatment was discontinued due to ineffectiveness and hematologic toxicity which did not allow to continue treatment (Figure 2).

The patient returned back to Moscow, where he underwent radiotherapy geared towards reduction of pain at N.N. Blokhin Russian Oncology Research Center in May 2015. Radiotherapy was carried out for the chest and thigh (24 Gys) and for the pelvis (19.5 Gys).

Unfortunately, the patient died from the disease progression on July 14, 2015.

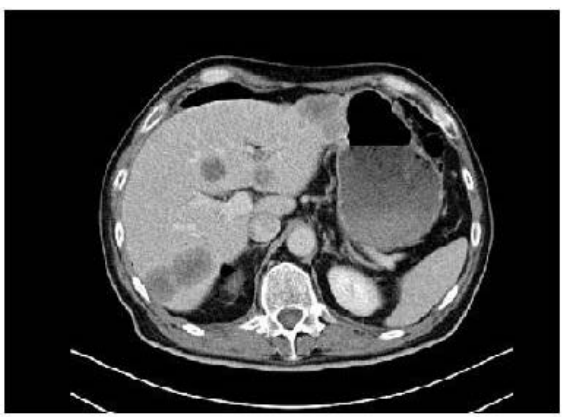

(a)

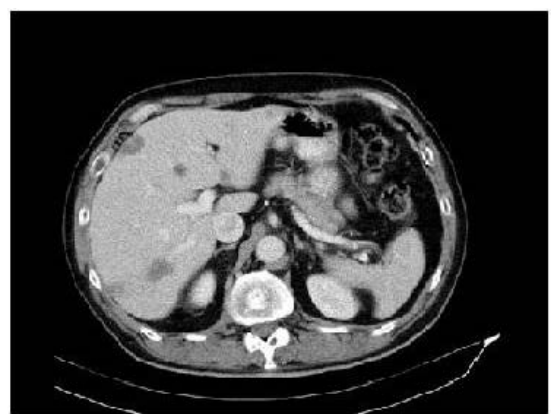

(b)

Figure 1. (a) CT scan of liver metastases prior to the treatment dated November 2013; (b) CT scan of liver metastases after 12 courses of intravenous FOLFIRINOX (combined with bevacizumab for the last 5 courses) dated June 2014 .

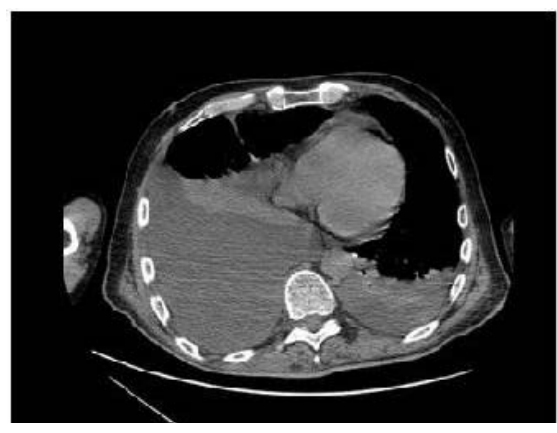

(a)

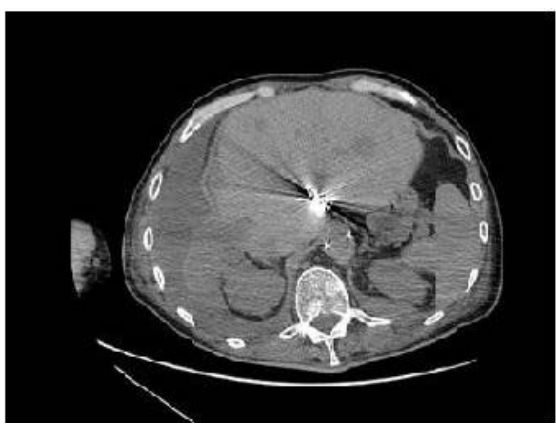

(b)

Figure 2. CT scans of bilateral pleurisy (a) and new liver metastases (b).

Table 1. Genetic analysis.

\begin{tabular}{ccccc}
\hline Tested Mutation & $\begin{array}{c}\text { Biopsy of } \\
\text { primary tumor } \\
21.11 .2013\end{array}$ & $\begin{array}{c}\text { Primary tumor } \\
\text { operation }\end{array}$ & $\begin{array}{c}\text { Mts in lymph } \\
\text { node }\end{array}$ & $\begin{array}{c}\text { Mts in liver } \\
\text { Operation } \\
16.07 .2014\end{array}$ \\
\hline $\begin{array}{c}\text { K-RAS } \\
\text { mutations } \\
\text { in the 2nd exon }\end{array}$ & $\mathrm{Wt}$ & $\mathrm{Wt}$ & $\mathrm{Wt}$ & $\begin{array}{c}\text { c.57 G } \rightarrow \mathrm{T} \\
\text { pLeu19Phe }\end{array}$ \\
$\begin{array}{c}\text { K-RAS } \\
\text { mutations } \\
\text { in the 3-d exon }\end{array}$ & $\mathrm{Wt}$ & $\mathrm{Wt}$ & & \\
\hline
\end{tabular}




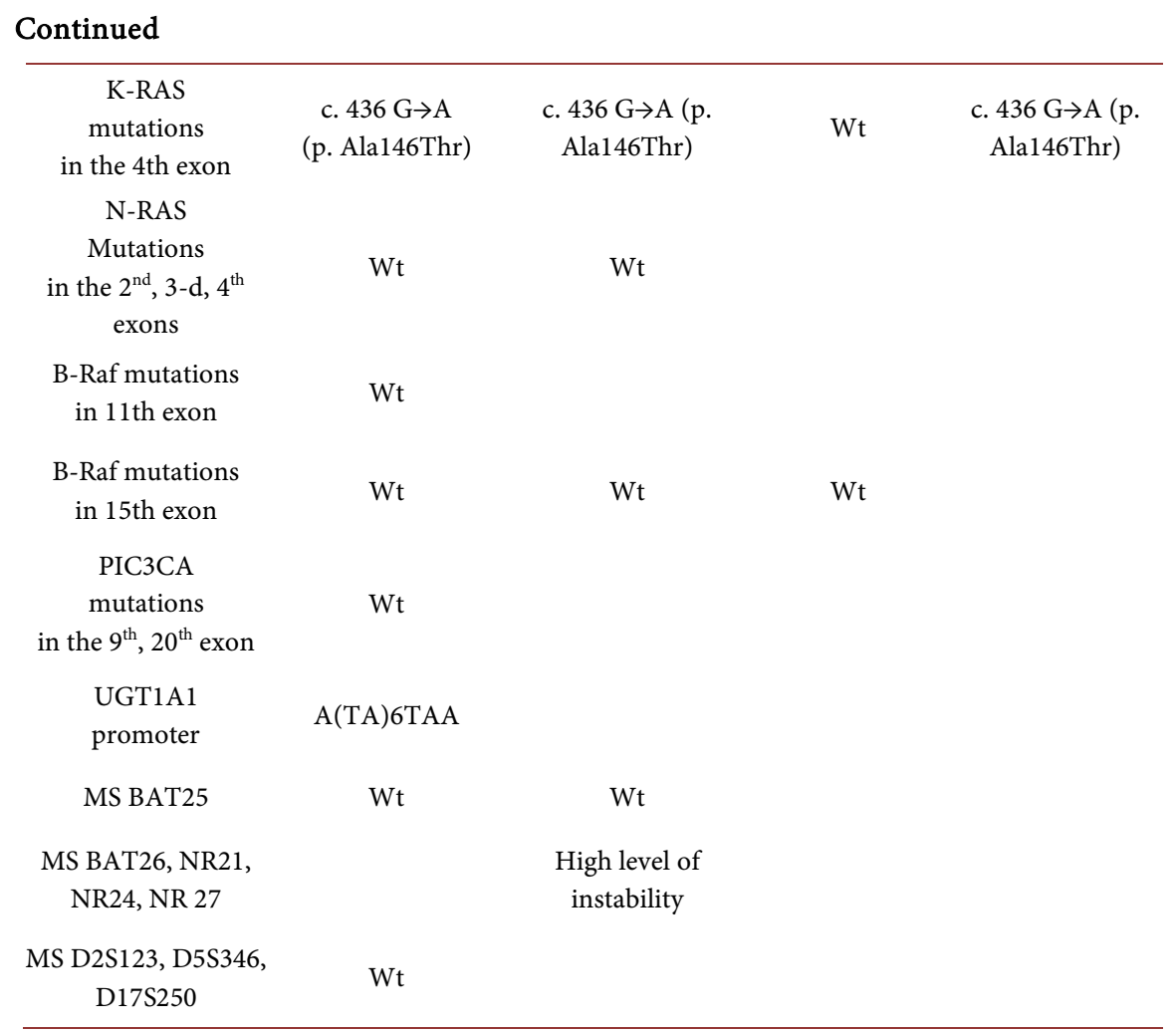

\section{Discussion}

It has been demonstrated here, that chronomodulated HAI triplet chemotherapy according to OPTILIV protocol was well tolerated and displayed high antitumor activity in this heavily-pretreated patient, allowing to achieve an objective response despite prior progression on FOLFIRINOX (the same triplet chemo by IV route) [14] [15]. This strategy permitted to overcome drug resistance and to perform further complete resection of the liver metastases with prolonged patient survival. The OPTILIV trial (irinotecan, 5-FU and oxaliplatin being given into the hepatic artery and cetuximab intravenously) is prospective international trial, whose primary end point is the conversion of initially uncontrolled and/or unresectable CLM to resection. The main end point was met, achieving a complete macroscopic resection rate of CLM in nearly $30 \%$ of all included patients with initially unresectable CLM [14]. Moreover, subgroup analysis suggested that the survival benefit from the liver-targeted chemo-surgery strategy was largely better in the patients receiving irinotecan, oxaliplatin and 5-FU as a chronomodulated hepatic artery infusion [16]. Several recent studies have reported that patients with right-sided primary tumours have worse survival outcomes and it is possible that these patients benefit less from standard therapies [17] [18] [19]. The study by Nanashima et al. reported in 2013 used old drugs, FU or CPT via the hepatic artery on 36 patients with colorectal liver metastases, 16 of whom had progressed after liver resection. Four of the patients had a complete response, 19 a partial response, for a total response rate of $64 \%$. The median survival was 32 months, with the complete responders having a median 
survival of 62 months [20]. In a recent study, the results from the phase-II clinical trial show that selected patients with strictly defined unresectable CRLM, despite pre-treatment with systemic chemotherapy and unfavorable oncologic characteristics, can experience enough response after combination treatment with HAI and systemic therapy to become resectable. Combination of HAI and systemic therapy resulted in very high response rates in both previously treated (72\%) and chemotherapy-naïve patients (82\%); overall 8\% CR (4 patients). The primary endpoint of the study (conversion to resection) was achieved with $47 \%$ of patients undergoing complete resection. Among the whole cohort, OS was 38 months, and one and 3-year survival rates were $92 \%$ and $55 \%$, respectively [3].

Despite the inefficiency of nivolumab in this case, initial analysis of nivolumab plus ipilimumab in patients with $\geq 6$ months follow-up demonstrated a manageable safety profile and clinical activity characterized by a $55 \%$ overall response rate and $80 \%$ disease control rate in patients with DNA mismatch repair-deficient (dMMR)/microsatellite instability-high (MSI-H) mCRC [21]. The ineffectiveness of nivolumab in this case may be due to the extensive heterogeneity in microsatellite unstable (MSI) between primary tumor and metastases, which has been recently shown in colorectal tumours [22]. Soimmunotherapy role even in patients with MSI status is not a landmark of response to anti-PD1 agents.

A pivotal phase 2 study by Le et al. highlighted the importance of mismatch-repair deficiency and/or (MSI-H) profiles in prediction of the clinical benefit of immune checkpoint blockade with pembrolizumab (anti-PD-1 drug) [23]. Moreover, the U.S. Food and Drug Administration (FDA) granted accelerated approval to anti-PD-1 drug pembrolizumab for adult and pediatric patients with unresectable/metastatic MSI-H/MMR deficient solid tumors (regardless the histotype) that have progressed following prior treatment and without satisfactory alternative treatment modalities. The approval also covered MSI-H CRC patients who progressed following treatment with a classic cytotoxic therapy (fluoropyrimidine, oxaliplatin, and irinotecan) [24].

Taken together, this literature review suggests that cure can be achieved in patients with initially unresectable CLM after the administration of the most effective downsizing chemotherapy, especially as first of second line. The patients with extensive unresectable CRLM, the majority of whom were previously treated, are able to undergo complete resection after HAI. Conversion to resection is associated with prolonged survival. The identification of optimum sequence of application of chronomodulated HAIC, immune checkpoint inhibitors, metastases surgery could increase the survival and possibly cure rate in these patients. Thus, chronomodulated HAI is useful in patients with liver metastases from colorectal cancer and deserves to be further assessed prospectively in clinical trials chemotherapy.

\section{Informed Consent}

Written informed consent was obtained from the next of kin of the patient for 
publication of this manuscript and any accompanying images.

\section{Conflicts of Interest}

The authors declare no conflicts of interest regarding the publication of this paper.

\section{References}

[1] Siegel, R., Naishadham, D. and Jemal, A. (2012) Cancer Statistics, 2012. CA: A Cancer Journal for Clinicians, 62, 10-29. https://doi.org/10.3322/caac.20138

[2] Scheele, J., Stangl, R. and Altendorf-Hofmann, A. (1990) Hepatic Metastases from Colorectal Carcinoma: Impact of Surgical Resection on the Natural History. British Journal of Surgery, 77, 1241-1246. https://doi.org/10.1002/bjs.1800771115

[3] D'Angelica, M.I., et al. (2015) Phase II Trial of Hepatic Artery Infusional and Systemic Chemotherapy for Patients with Unresectable Hepatic Metastases from Colorectal Cancer: Conversion to Resection and Long-Term Outcomes. Annals of Surgery, 261, 353-360. https://doi.org/10.1097/SLA.0000000000000614

[4] Adam, R., Laurent, A., Azoulay, D., et al. (2000) Two-Stage Hepatectomy: A Planned Strategy to Treat Irresectable Liver Tumors. Annals of Surgery, 232, 777-785. https://doi.org/10.1097/00000658-200012000-00006

[5] Chang, A.E., Schneider, P.D., Sugarbaker, P.H., et al. (1987) A Prospective Randomized Trial of Regional versus Systemic Continuous 5-Fluorodeoxyuridine Chemotherapy in the Treatment of Colorectal Liver Metastases. Annals of Surgery, 206, 685-693. https://doi.org/10.1097/00000658-198712000-00001

[6] Kemeny, N., Daly, J., Reichman, B., et al. (1987) Intrahepatic or Systemic Infusion of Fluorodeoxyuridine in Patients with Liver Metastases from Colorectal Carcinoma. A Randomized Trial. Annals of Internal Medicine, 107, 459-465. https://doi.org/10.7326/0003-4819-107-4-459

[7] Kemeny, N.E., Niedzwiecki, D., Hollis, D.R., et al. (2006) Hepatic Arterial Infusion versus Systemic Therapy for Hepatic Metastases from Colorectal Cancer: A Randomized Trial of Efficacy, Quality of Life, and Molecular Markers (CALGB 9481). Journal of Clinical Oncology, 24, 1395-1403.

https://doi.org/10.1200/JCO.2005.03.8166

[8] Bouchahda, M., Adam, R., Giacchetti, S., et al. (2009) Rescue Chemotherapy Using Multidrug Chronomodulated Hepatic Arterial Infusion for Patients with Heavily Pretreated Metastatic Colorectal Cancer. Cancer, 115, 4990-4999.

https://doi.org/10.1002/cncr.24549

[9] Allard, M.A., Sebagh, M., Baillie, G., et al. (2015) Comparison of Complete Pathologic Response and Hepatic Injuries between Hepatic Arterial Infusion and Systemic Administration of Oxaliplatin in Patients with Colorectal Liver Metastases. Annals of Surgical Oncology, 22, 1925-1932.

https://doi.org/10.1245/s10434-014-4272-7

[10] Wicherts, D.A., de Haas, R.J., et al. (2009) Patients with Initially Unresectable Colorectal Liver Metastases: Is There a Possibility of Cure? Journal of Clinical Oncology, 27, 1829-1835.

[11] Levi, F. and Schibler, U. (2007) Circadian Rhythms: Mechanisms and Therapeutic Implications. Annual Review of Pharmacology and Toxicology, 47, 593-628.

https://doi.org/10.1146/annurev.pharmtox.47.120505.105208 
[12] Giacchetti, S., Bjarnason, G., Garufi, C., et al. (2006) Phase III Trial Comparing 4-Day Chronomodulated Therapy versus 2-Day Conventional Delivery of Fluorouracil, leucovorin, and Oxaliplatin as First-Line Chemotherapy of Metastatic Colorectal Cancer: The European Organisation for Research and Treatment of Cancer Chronotherapy Group. Journal of Clinical Oncology, 24, 3562-3569.

https://doi.org/10.1200/JCO.2006.06.1440

[13] Giacchetti, S., Dugué, P.A., Innominato, P.F., et al. (2012) Sex Moderates Circadian Chemotherapy Effects on Survival of Patients with Metastatic Colorectal Cancer: A Meta-Analysis. Annals of Oncology, 23, 3110-3116.

https://doi.org/10.1093/annonc/mds148

[14] Levi, F., Karaboué, A., Etienne-Grimaldi, M.C., Paintaud, G., et al. (2017) Pharmacokinetics of Irinotecan, Oxaliplatin and 5-Fluorouracil during Hepatic Artery Chronomodulated Infusion. A Translational European OPTILIV Study. Clinical Pharmacokinetics, 56, 165-177. https://doi.org/10.1007/s40262-016-0431-2

[15] Levi, F., et al. (2016) Conversion to Resection of Liver Metastases from Colorectal Cancer with Hepatic Artery Infusion of Combined Chemotherapy and Systemic Cetuximab in Multicenter Trial OPTILIV. Annals of Oncology, 27, 267-274. https://doi.org/10.1093/annonc/mdv548

[16] Bouchahda, M., Boige, V., Smith, D., et al. (2016) Early Tumor Response as a Survival Predictor in Previously-Treated Patients Receiving Triplet Hepatic Artery Infusion and Intravenous Cetuximab for Unresectable Liver Metastases from Wild-Type KRAS Colorectal Cancer. European Journal of Cancer, 68, 163-172.

[17] Petrelli, F., Tomasello, G. and Borgonovo, K. (2017) Prognostic Survival Associated with Left-Sided vs. Right-Sided Colon Cancer: A Systematic Review and Meta-Analysis. JAMA Oncology, 3, 211-219. https://doi.org/10.1001/jamaoncol.2016.4227

[18] Tejpar, S., Stintzing, S. and Ciardiello, F. (2017) Prognostic and Predictive Relevance of Primary Tumor Location in Patients with RAS Wild-Type Metastatic Colorectal Cancer: Retrospective Analyses of the CRYSTAL and FIRE-3 Trials. JAMA Oncology, 3, 194-201. https://doi.org/10.1001/jamaoncol.2016.3797

[19] Venook, A.P., Niedzwiecki, D. and Innocenti, F. (2016) Impact of Primary $\left(1^{\circ}\right)$ Tumor Location on Overall Survival (OS) and Progression-Free Survival (PFS) in Patients (Pts) with Metastatic Colorectal Cancer (mCRC): Analysis of CALGB/SWOG 80405 (Alliance). American Society of Clinical Oncology, 34, Article ID: 3504. https://doi.org/10.1200/JCO.2016.34.15_suppl.3504

[20] Nanashima, A., et al. (2013) Recent Advances and Significance of Intra-Arterial Infusion Chemotherapy in Non-Resectable Colorectal Liver Metastasis. Journal of Gastrointestinal Oncology, 4, 164-172.

[21] Overman, M.J., McDermott, R., Leach, J.L., et al. (2017) Nivolumab in Patients with Metastatic DNA Mismatch Repair-Deficient or Microsatellite Instability-High Colorectal Cancer (Check Mate 142): An Open-Label, Multicentre, Phase 2 Study. The Lancet Oncology, 18, 1182-1191. https://doi.org/10.1016/S1470-2045(17)30422-9

[22] Sagaert, X., Tejpar, S., Desmedt, L., et al. (2017) Intratumoral Heterogeneity in Colorectal Cancer: Can Histology Be Used as a Guidance for Molecular Testing? Journal of Clinical Oncology, 35, 611.

[23] Le, D.T., Uram, J.N., Wang, H., Bartlett, B.R., Kemberling, H., Eyring, A.D., et al. (2015) PD-1 Blockade in Tumors with Mismatch-Repair Deficiency. The New England Journal of Medicine, 372, 2509-2520. https://doi.org/10.1056/NEJMoa1500596

[24] Vranić, S. (2017) Microsatellite Instability Status Predicts Response to Anti-PD-1/PD-L1 Therapy Regardless the Histotype: A Comment on Recent Advances. Bosnian Journal of Basic Medical Sciences, 17, 274-275. 